\title{
Low-Atomic-Number Nanometric Film Production Method for keV Electron Scattering Measurements
}

\author{
Mordechai Geller ${ }^{1}$ and Itzhak Orion ${ }^{2}$ \\ 1. Nuclear Research Center-Negev (NRCN), Beer-Sheva 84190, Israel \\ 2. Department of Nuclear Engineering, Ben-Gurion University of the Negev, Beer-Sheva 84105, Israel
}

\begin{abstract}
Attenuating mediums, targets and barriers made of submicronic layers with low average atomic number (low Z) and minimal electronic density that reduces the elastic scattering and absorption of radiation are required for many applications. This work describes the development and characterization of submicronic Lexan (Polycarbonate) polymer foils with low $\mathrm{Z}$ for two new electron inelastic mean free path assessment methods. Lexan layers with thicknesses of $120 \mathrm{~nm}$ to $240 \mathrm{~nm}$ were developed and fabricated using spin coating. The submicronic layers were characterized by AFM and CSI for thickness, roughness and levelness. Roughness was found to be $1.0-2.4 \mathrm{~nm}$ rms, and the change in total thickness was within $\pm 7.5 \%$. The results of total current measurements using $177 \mathrm{~nm}$ Lexan foil irradiated under an SEM electron beam were compared to those for a similar polymer foil. The first step of a wide spectrum method experiment was performed at the ESRF using Lexan submicronic layers on a silicon substrate. The signal peak and the multiple inelastic scattering peak of the Lexan spectrum was similar to those previously measured on carbon films. This study supported the suitability of the developed Lexan films for electron scattering measurements.
\end{abstract}

Key words: HAXPES, scattering, layer, SEM, spin-coating.

\section{Introduction}

The IMFP (inelastic mean free path) of an electron in a solid is an important factor in quantitative surface analysis methods such as AES and XPS that measure the scattering or ejection of the electron from a solid surface. The value of the IMFP is used, together with the experimental setup, to determine the mean depth of the solid surface being analyzed via a certain method. It is also needed to calculate single electron transport in solids [1, 2]. Likewise, the IMFP is used to set the surface sensitivity in other surface characterization methods based on the emission or ejection of electrons from solid surfaces, such as low-energy electron diffraction and UV photoelectron spectroscopy among others, for which information about the low energy electron transport parameter in the solid is needed [3].

Corresponding author: Itzhak Orion, Ph.D. in physics and nuclear engineering, head of the nuclear engineering department, main research field: radiation transport.
The electron IMFP depends both on matter and on electron energy and its value typically range is from 1 to 20 crystal lattice spacing (3-50 $\AA$ ) for electron energies of anywhere from 10 to $2,500 \mathrm{eV}$, which is the practical range for XPS and AES. Although the electron IMFP is considered a bulk property, in certain applications IMFP surface corrections can be applied. The IMFP can be calculated and measured, but the numerous uncertainty factors render reliable data difficult to obtain.

An approximation of IMFP energy dependency can be found in Refs. [3, 4]: $\lambda_{(E)}=k E^{p}$, where $k$ and $p$ are fit coefficients.

There are three accepted IMFP evaluation methods:

(1) Optic experiments data used to calculate for a limited number of substances;

(2) Backscattered electron intensity measured for electrons ejected from the surface, e.g., EPES (elastic-peak electron spectroscopy), for several solid elements;

(3) Prediction formula used in calculations for all substances. 
The first two IMFP evaluation methods are considered to be more effective than the third method listed above [2, 3, 5]. All three methods are characterized by uncertainties that affect and impair IMFP evaluation.

All experimental methods use thin, submicronic layers irradiated with electrons, or photons to generate emitted electrons. Among the main uncertainties in IMFP assessments are the precision of the thickness measurement and the surface characterization of the layer. Indeed, increases in layer thickness or decreases in electron energy are accompanied by the weakening or almost complete disappearance of the electron signal peak. Although the first two methods in the list above attempt to circumvent such uncertainties, they still depend on well-defined, submicronic layers with proper thickness.

Among the first methods used to evaluate the IMFP was Over-layer [3, 6-9], which has two main drawbacks. First, experiments using the over-layer approach suffer from large uncertainties due to non-uniform thickness of the film layer and unreliable film thickness measurements. Second, the over-layer method relies on the basic assumption that elastic scattering is negligible, and as such, electrons are calculated as moving in a straight line from their origin to the surface.

Currently, the principal method used to evaluate IMFP is EPES (elastic-peak electron spectroscopy) [3, 5]. The reliability of this method, however, depends on an evaluation of the electron elastic mean free path length for a given solid angle and energy, which is itself not well defined because the elastic scattering occurs mainly in a forward direction. Indeed, because the overall elastic cross-section depends strongly on the electron-atom interaction potential, it is generally not recommended that the electron elastic mean free path length be used to describe electron transport [10]. In both the over-layer and elastic-peak methods, increasing the electron energy decreases the elastic backscattered electron intensity, a fact that limits the higher electron energies in such measurements. To overcome such energy limits, a new method of electron IMFP evaluation is essential.

Two methods of analyzing the IMFP of electrons at energies above $5 \mathrm{keV}$ include the TCM (total current measurements) $[10,11]$ and the WES (wide energy spectrum) methods [12]. Tests to experimentally inspect the validities of the two methods require that a submicronic layer or film be manufactured from the sample being tested. The ability to fabricate controlled and high quality layers with known thicknesses is imperative for the validation of any method.

This paper presents a submicronic layer fabrication and characterization method based on low atomic-number materials to facilitate the study and measurement of electron transport properties using the total current measurements and wide spectrum new methods.

\subsection{TCM (Total Current Measurements) Method}

Based on the same principle as EPES, the TCM method exploits the indirect effect of inelastic interactions on elastic interactions to measure the influence of directional deviation on the energy loss. However, in contrast to the requirement in EPES that the full spectrum be measured, only the electron beam current is measured in TCM. Thus, the electron beam penetrates a thin film with a known thickness and total current attenuation is measured. At a certain energy level, measurements must be made in pairs: the initial current $\mathrm{I}_{0 \mathrm{t}}$ and the total electron current that passed through the film. For each electron beam energy, the authors obtain the measured current ratio:

$$
\text { measured current ratio }=\frac{\sum \frac{\mathrm{dI}}{\mathrm{dE}}}{\mathrm{I}_{0 \mathrm{t}}}
$$

Because the authors are looking for inelastic scattered electrons, the beam must be measured at a narrow solid angle, and therefore, no wide-angle scattered electrons will be detected. This method is 
built on the basic assumption that elastic scattering processes and inelastic energy processes can be separated based on the differences in their angular dependence values. The counting contribution of elastic interaction occurs mainly for large angles, while the change in momentum in the inelastic interactions is insignificant. Hence, current measurements along the initial electron path should reveal the inelastic electron scattering dependence. Using the measured current ratios, the electron IMFP can be analyzed by the calibration graph of the total current attenuation versus IMFP.

\subsection{WES (Wide Energy Spectrum) Method}

Also reliant on an algorithm for evaluating layer thickness, this new WES method is based on an average energy shift analysis of electrons that was developed by our group and published in 2012 [13]. The WES method exploits the attenuated electron peak counts in XPS (X-ray photoelectron spectroscopy) rather than use the signal electron peaks emitted from the sample (due to photon beam irradiation) and then calculate the mean electron energy loss.

When analyzing thin layer on substrate, the signal electron peak in the spectrum is resulted from attenuating electrons within the layer at the same energy as the initial kinetic energy. In electron spectroscopy, the signal electron peak can usually be separated from the scattered electrons. Indeed, a lot of work has been invested in recent years to develop the ability to separate this peak [4]. In thicker layers, the main cause of electron attenuation is MIC (multiple inelastic collisions) [12], for which HAXPES (hard $\mathrm{X}$-ray photoelectron spectroscopy) is essential. Using HAXPES in third generation synchrotrons enables the electron spectrum to be extended up to $15 \mathrm{keV}$ compared to only $2 \mathrm{keV}$ under the common XPS method.

The probability of electron energy loss in an individual collision is almost independent of the initial electron energy [4]. To analyze the IMFP for a certain material, several samples of that material with known thicknesses and having the same electron beam energy must be measured. Detailed calculations of the extraction of the IMFP of carbon were published in Ref. [12] using Eqs. (1-3) therein. In this work, the authors show for the first time experimental spectrum for Lexan to ascertain the WES method implementation.

\section{Materials and Method}

\subsection{Fabrication and Characterization of Lexan Thin Film}

Film thickness has a significant influence on the interactions and paths of the electrons in the TCM experiment. Because at energies of 5-15 keV electron interactions readily take place in films with thicknesses of a few hundred nanometers, film thickness should therefore be in the range of 100-250 $\mathrm{nm}$. Moreover, the film must be continuous and of uniform thickness to prevent thickness deviation errors. Therefore, overall film thickness uniformity was set at a maximum of $\pm 7.5 \%$ from the nominal and overall roughness was set at no more than $10 \pm 1$ nm rms.

The thin film fabrication technique developed in this study enables the fabrication (on a glass substrate) and characterization of uniform, ultrathin films of Lexan (polycarbonate) with thicknesses from $110 \mathrm{~nm}$ to $330 \mathrm{~nm}$. After fabrication and characterization, the film is then transferred to measuring window targets 2-6 $\mathrm{mm}$ in diameter. This method has also been used successfully to coat a $10 \mathrm{~mm}^{2}$ and $8 \mathrm{~mm}^{2}$ rectangular $\mathrm{Cu}$ and Si substrate with Lexan films as thin as $50 \mathrm{~nm}$. The overall rms surface roughness of the film fabricated for the current study was 9-11 nm due to the surface waviness typical of Lexan films, while its local rms surface roughness was $1.0-2.4 \mathrm{~nm}$. The maximum deviation from nominal film thickness was $\pm 7.5 \%$.

Spin coating was chosen as the preferred fabrication method for the window targets for the TCM and WES 
experiments. Widely used in thin film fabrication on a variety of different substrates, the spin coating technique [14] is the preferred method of applying a thin, uniform film to a flat substrate. The typical thickness range of a film fabricated by spin coating is 1-200 $\mu \mathrm{m}$. Films whose thicknesses are less than 200 $\mathrm{nm}$ are considered ultra-thin and the process of fabricating them by spin coating has not been fully explored in the field.

The spin coating process basically entails dropping a solution of dissolved solid material, such as a polymer, on a flat, spinning substrate. The centrifugal force applied to the solution causes it to flow and spread across the substrate, and eventually, some of the solution detaches from the substrate, leaving a very thin layer behind. The final thickness and other properties of the resultant film are affected both by the solution characteristics, such as its viscosity and concentration, and by the spinning parameters, including the acceleration, speed and duration of the spinning. In most cases, film thickness depends primarily on two independent variables: solution concentration and spinning speed [15]:

$$
h_{f} \propto c_{0}^{2} \omega^{-0.5}
$$

where, $c_{0}$ is the solution's initial concentration, $\omega$ is the spin speed, and the initial viscosity is constant. Keeping all parameters, including solution concentration, constant, film thickness depends only on the spin speed:

$$
h_{f} \propto \omega^{-a}
$$

Lexan is constructed from carbon, oxygen and hydrogen atoms and is a low atomic number (low Z) material. Insofar as it dissolves easily and is sufficiently strong, Lexan is a good candidate for use in the construction of a self-supported, ultrathin film.

The Lexan solution for this study was made by dissolving $0.2 \mathrm{~g}$ Lexan with $6 \mathrm{~cm}^{3}$ 1,2-dichloroethane and $4 \mathrm{~cm}^{3}$ dichloromethane in a clean glass flask for a $1.56 \mathrm{wt} \%$ solid concentration. The surface of a clean microscope slide was used as a substrate and fitted in a spin coater. The glass substrate was then rotated at various speeds from 2,000 rpm to 4,500 rpm. Once the glass slide was spinning steadily at a given speed, 30 $\mu \mathrm{L}$ of Lexan solution was dropped at a uniform rate by micropipettor from a height of $15 \mathrm{~mm}$ on the center of the spinning glass substrate, ensuring that the solution falls perpendicular to the spinning glass. The glass substrate was left to turn at a constant speed for three minutes and then placed on a hot plate to dry for three more minutes at $40{ }^{\circ} \mathrm{C}$. All these actions were carried out in a well-ventilated hood in a climate-controlled laboratory $\left(22-26{ }^{\circ} \mathrm{C}, \quad 28-35 \%\right.$ humidity). The ultrathin film fabricated on the glass substrate was then peeled off the glass and transferred to aluminum disks, each with a window that measured 2-6 $\mathrm{mm}$ in diameter.

Once the authors mastered the film fabrication process and established a consistent procedure, the authors fabricated several Lexan films on glass substrates. The films were fabricated in groups, for each of which the spinning speed, solution concentration and quantity of solution were held constant. Among groups, only the spinning speed was changed. Under these experimental conditions, we found that the minimum spinning speed at which a film can be fabricated with the desired physical properties is $1,500 \mathrm{rpm}$. Likewise, the maximum speed at which a film can be fabricated and still be thick enough to be transferred to a window is about 5,000 rpm. Changing other parameters, such as the solution solid content, should affect those limits.

Analyses of the resultant thickness values versus the spinning speeds revealed a deviation of $\pm 5 \%$. The systematic error in spinning speed measurements was $\pm 5 \%$. Thickness measurements were therefore averaged to reduce inaccuracy and spread.

Starting with an initial Lexan solution concentration of $1.56 \mathrm{wt} \%$, Lexan films of several thicknesses were produced at different spinning speeds. Thickness data were fit by power regression to calculate the desired film thickness with good precision $( \pm 2 \%)$ :

$$
h_{f}(n m)=15545 \omega^{-0.573}(\omega \text { in rpm }) .
$$


A second prediction curve was made with a power of -0.5 , as noted by Lawrence, Extrand, Hall and others [15-17]. Although the second curve under- or over-predicted thickness for lower or higher rpm disk speeds, respectively, the overall error still remained well within the $\pm 6 \%$ range (data and prediction curves summarized in Fig. 1).

$$
h_{f}(n m)=8860 \omega^{-0.5}(\omega \text { in rpm }) \text {. }
$$

Film thickness properties, including average thickness, roughness, levelness and continuity, were measured, observed and analyzed. Film thickness was measured primarily by AFM (atomic force microscopy) and was cross correlated on some samples by interferometry and by Ellipsometry. The AFM measurements (Veeco Dimension 3100 SPM) were done at a scanning length of $40 \mu \mathrm{m}$, a vertical resolution of $1 \mathrm{~nm}$ and a lateral resolution of $5 \mathrm{~nm}$. Over 25 different films were measured, each one at least twice and some three or four times at different locations over the surface of the film (Fig. 2).

Interferometry measurements (Zygo NEWVIEW 200 interferometer) covered a scanning area of $0.2 \mathrm{~mm} \times 0.3 \mathrm{~mm}$ at a vertical resolution of $0.1 \mathrm{~nm}$. Ellipsometry measurements (Sentech SE 800 spectroscopic ellipsometer) were carried out at a $70^{\circ}$ AOI (angle of incidence) or at MAI (multiple angles of incidence) in the $55^{\circ}$ to $70^{\circ}$ range. Thickness measurements recorded with AFM were found to be within the limit of $\pm 5 \%$ of the same measurements by interferometry, and they were a maximum of $6 \%$ larger than the same measurements by Ellipsometry. Surface roughness was measured by AFM with a window of $60 \times 60 \mu \mathrm{m}^{2}$ (Fig. 3) and by interferometry with a window of $200 \times 200 \mu \mathrm{m}^{2}$ (Fig. 4). Surface mean roughness $(\mathrm{Ra})$ measurements using the former method were in the range of 1.0-2.4 nm rms, but using the interferometer with its wider window, they were $10 \pm 1 \mathrm{~nm}$ rms. This discrepancy is due to the inability of the small window area of the AFM to capture the film's typical waviness (Fig. 4).

With the AFM and interferometry, film uniformity and levelness within the specific window boundaries can be observed. In all tests (over 25 tests), films were found to be continuous and to be free of holes. Moreover, at radial distances of at least $10 \mathrm{~mm}$ from the centers of tested films, film thicknesses changed by a maximum of $10 \%$. When the target window diameter was $6 \mathrm{~mm}$, the maximum change observed in thickness was 6\%.

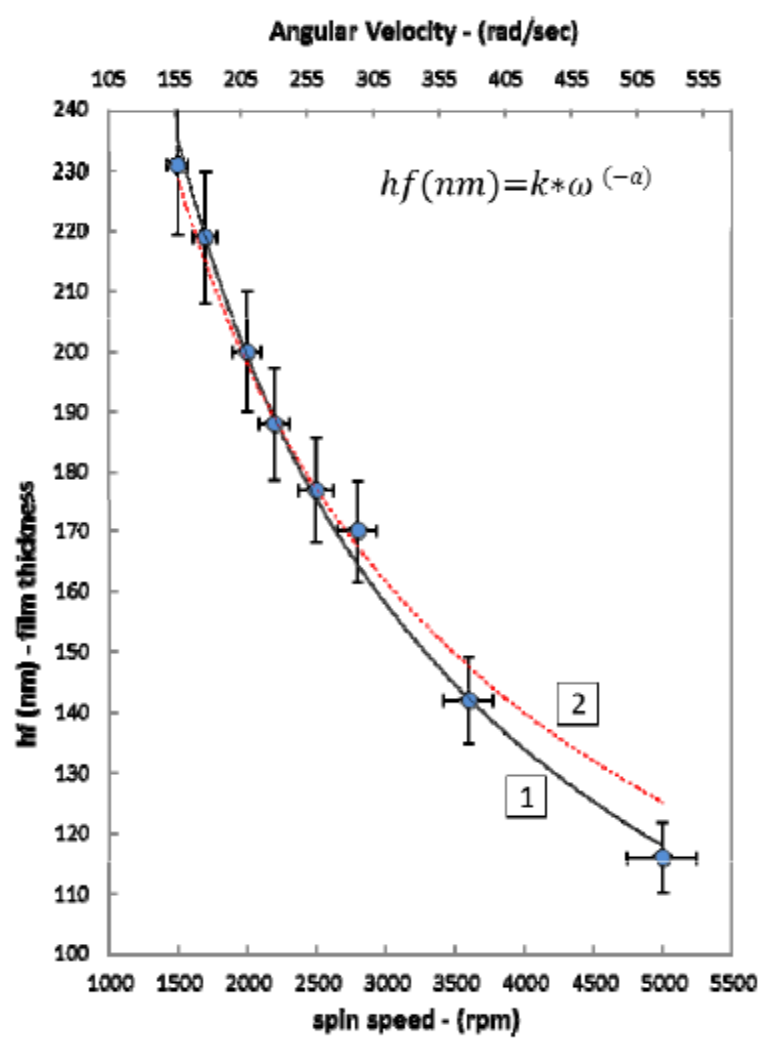

Fig. 1 Average Lexan film thickness, $h_{f}$ vs. spin speed @ Initial 1.56 wt. \% Lexan solution concentration: Line 1: a = 0.573, $k=15,545, R^{2}=0.995$; Line 2: $a=0.5, k=8,860, R^{2}=$ 0.985 .

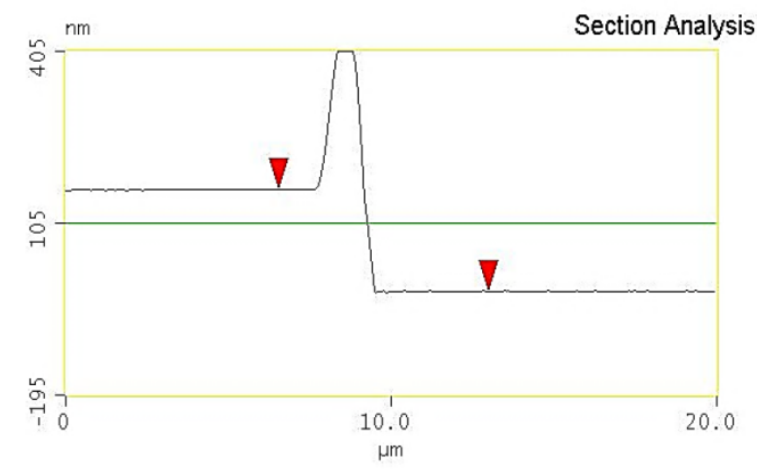

Fig. 2 AFM thickness analysis of Lexan film. 


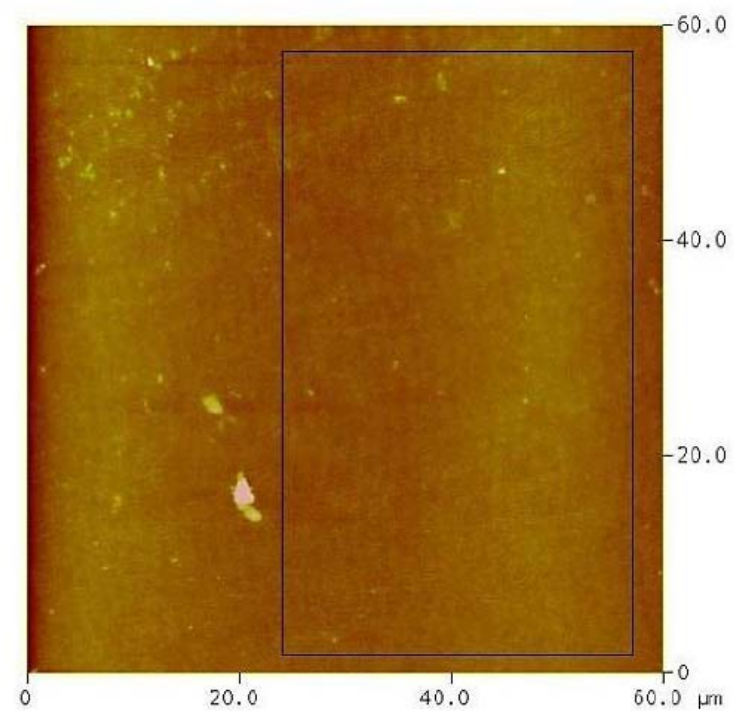

Fig. 3 Lexan surface roughness analysis using AFM.

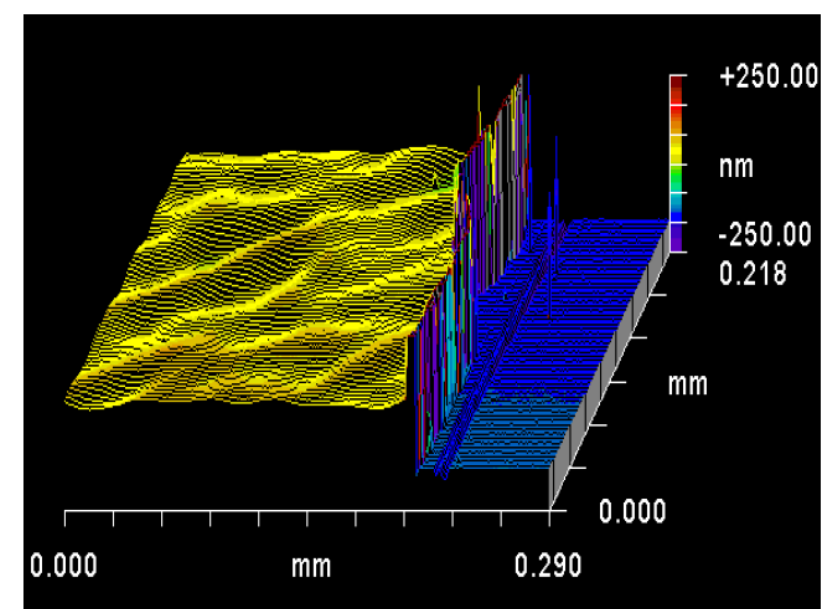

Fig. 4 Interferometer mapping image of Lexan film surface.

\section{Experimental}

\subsection{Wide Spectrum in HAXPES}

The use of HAXPES (hard X-ray photoelectron spectroscopy) in third generation synchrotrons $[9,18]$ facilitated photoelectron energy as high as $15,000 \mathrm{eV}$. The HAXPES is located at the Spanish beam-line (Spline) in the ESRF (European Synchrotron Radiation Facility). More details about the experimental setup are available in Refs. [19, 20]. In the HAXPES setup, the 1-s shell electrons of the silicon substrate were used to generate photoelectron emission. Lexan film $\left(9 \times 9 \mathrm{~mm}^{2}\right.$ and $97.5 \mathrm{~nm}$ thick $)$ on silicon (1 00 ) bulk was used with monochromatic photon beams at $10 \mathrm{keV}$ and $14 \mathrm{keV}$.

\subsection{TCM (Total Current Method)}

An electron gun (Quanta 200 SEM, Philips) with a well-defined high-voltage range (3-20 kV) and emission current values of 100-120 $\mu \mathrm{A}$ that produce a collimated coherent electron flux of 40-7,000 pA was used.

To measure the attenuated flux of electrons that penetrated the film, a special apparatus was built (Fig. 5). Two identical brass Faraday cups were soldered to a copper plate. The inclusion of Faraday cups ensured that secondary or backscattered electrons would not affect current measurements. Each cup measured 9 $\mathrm{mm}$ in diameter by $10 \mathrm{~mm}$ in height, and it had a hole in its top that was $1 \mathrm{~mm}$ in diameter. The $200 \mathrm{~nm}$ attenuation layer was isolated by a ceramic insulating ring and placed on top of one of the Faraday cups. The SEM step-motor that controls sample position was detached and its plug was used to connect the electrometer BNC cable to the Faraday cup that collected the electrons penetrating the tested layer, inside the SEM cavity. At its other end, this plug was connected to the electrometer to allow us to measure currents from the Faraday cups. A grounding wire running from the top layer to the stage ensured that any electrons that accumulated there would be discharged.

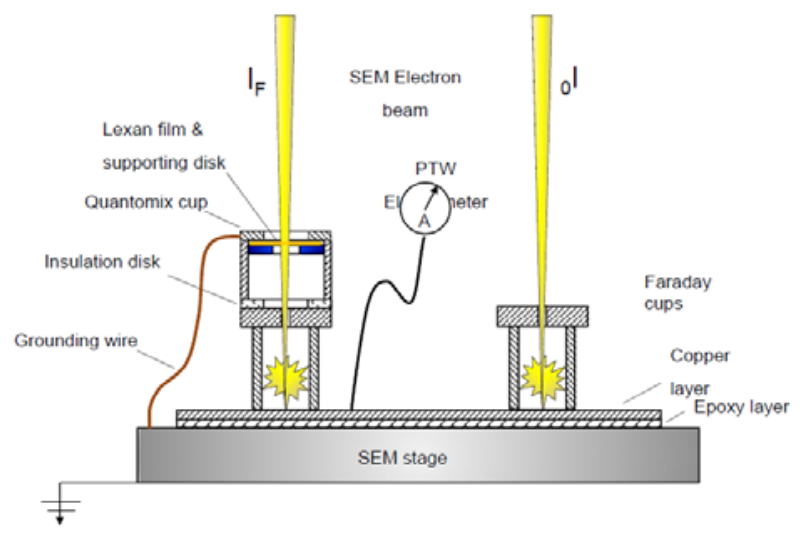

Fig. 5 Experimental setup for SEM total current method. 
The currents from the Faraday cups were measured using an electrometer (PTW UNIDOS). The current measured from the cup fitted with the Lexan film attenuation layer is $\mathrm{I}_{\mathrm{F}}$ and that measured from the second, bare cup is $\mathrm{I}_{0}$. All experiments were conducted under HV (high vacuum) at 4.2 micro-Torr to prevent beam dispersion using a spot size of 7.0, a filament current of $2.68 \mathrm{~A}$ and an emission of $94 \mu \mathrm{A}$. The SEM was operated in a Raster mode of $400 \times 400$ $\mu \mathrm{m}^{2}$. For each electron energy, the beam was shifted from the covered cup to the bare one to obtain $\mathrm{I}_{\mathrm{F}}$ and $\mathrm{I}_{0}$, respectively. The electron beam energy was set in several values between $3 \mathrm{keV}$ and $15 \mathrm{keV}$ for each pair. Film thickness uniformity precision was $\pm 7.5 \%$ of the nominal, and local roughness was $1.0-2.4 \mathrm{~nm}$ rms.

\section{Results}

\subsection{Wide Spectrum in HAXPES}

Results from the wide spectrum method were obtained for Lexan film with a thickness of $97.5 \mathrm{~nm}$ on silicon bulk. The spectrum was found to be similar to previous results on carbon films [12]. In Fig. 6, the photoelectron signal peak appears at energy of 12,168 $\mathrm{eV}$ (SP), while the wide peak (MIC) at lower energies is due to multiple inelastic electron collisions in the Lexan film. The spectrum indicated that the wide peak can be measured not only on pure elements, but also on dedicated, thin film polymers.

\subsection{Total Current Method}

In the TCM experiment, seven points were measured at energies of 1-15 keV using an SEM. The current was of negative value and measured in units of pA (pico-Ampere). Maximum current fluctuations were $\pm 50 \mathrm{pA}$ in all measurements except that at 3 $\mathrm{keV}$ energy, for which the current fluctuated $5 \mathrm{pA}$, and that at $1 \mathrm{keV}$, for which the current fluctuation was a steady $0 \mathrm{pA}$. Because the equipment reading error was $\pm 0.5 \%$, the $\mathrm{I}_{\mathrm{F}} / \mathrm{I}_{0}$ ratio error was constrained by $\pm 3 \%$. The results of the Lexan TCM experiment are shown in Table 1. A comparison with the results of the previously performed TCM experiment on Quantomix ${ }^{\circledR}$ films is shown in Fig. 7. The similarity between the two experiments results indicates that the electron transport properties in the developed Lexan film are similar to those of the Quantomix film. An analysis of the results reveals a major difference between the initial measured currents of the Lexan and Quantomix experiments, the former of which were about 15 times higher. The higher measured currents are due to setup improvements that led to a reduction in the resistance of the connectors. The higher measured current increased the sensitivity of the measurements, enabling them to be performed at low energies of $3 \mathrm{keV}$ and $1 \mathrm{keV}$.

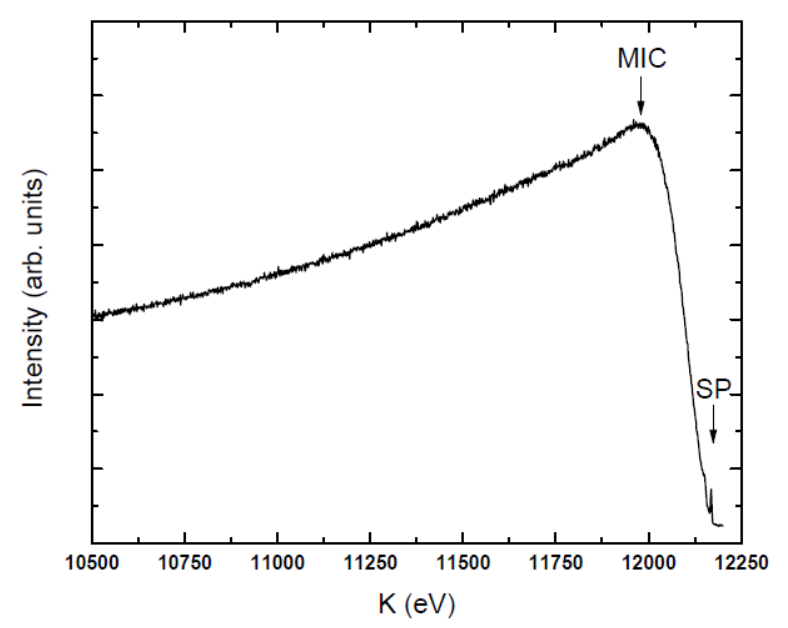

Fig. 6 MIC peak in Lexan 100 nm layer.

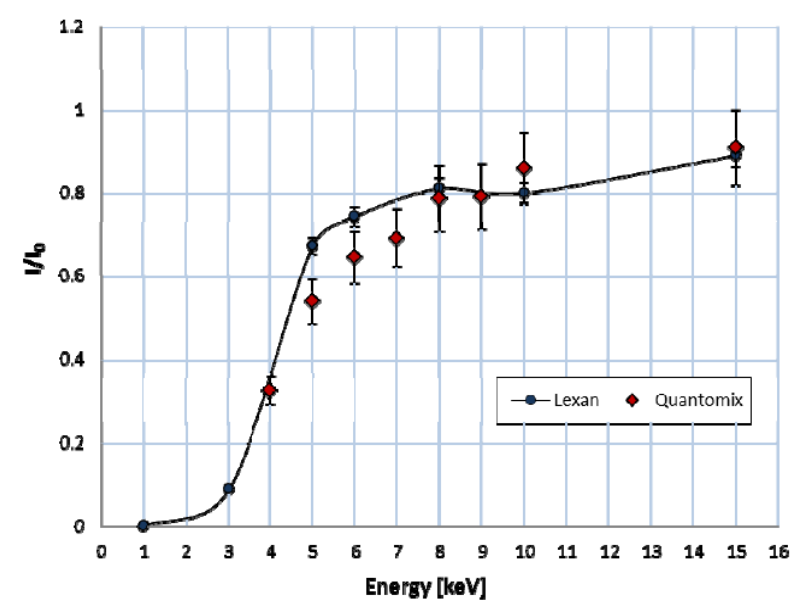

Fig. 7 Experimental results of SEM total current measurements for Lexan and Quantomix films. 
Table 1 The Lexan film total current method measurements results using electrometer.

\begin{tabular}{llll}
\hline Energy $[\mathrm{keV}]$ & $\begin{array}{l}\mathrm{I}_{0} \\
{[\mathrm{pA}]}\end{array}$ & $\begin{array}{l}\mathrm{I}_{\mathrm{F}} \\
{[\mathrm{pA}]}\end{array}$ & $\mathrm{I}_{\mathrm{F}} / \mathrm{I}_{0}$ \\
\hline Error & $\pm 50 \mathrm{pA}$ & $\pm 50 \mathrm{pA}$ & $\pm 3 \%$ \\
1 & $-3,950$ & 0 & 0.00 \\
3 & $-3,930$ & $-346 \pm 5$ & 0.09 \\
5 & $-4,230$ & $-2,840$ & 0.67 \\
6 & $-4,790$ & $-3,550$ & 0.74 \\
8 & $-4,840$ & $-3,920$ & 0.81 \\
10 & $-5,010$ & $-4,000$ & 0.80 \\
15 & $-6,800$ & $-6,040$ & 0.89 \\
\hline
\end{tabular}

The film withstands the transfer from the high vacuum $\left(\sim 4.10^{-6}\right.$ Torr $)$ to the air environment in the SEM. The film endured the irradiation of a $10-\mathrm{kV}$ electron beam spot-size of 1.5 current of about $7 \mathrm{nA}$ during $3 \mathrm{~min}$. Note that although measurements for the Lexan film taken at the lower energies exposed the film to higher temperatures compared to the Quantomix film, the Lexan film did not exhibit any damage.

\section{Conclusion}

The ability to develop and fabricate a submicronic attenuating film from a low atomic number polymer for the measurement and evaluation of electron IMFP in thin layers was studied. The Lexan polymer was found to be suitable compound for the preparation of thin films using the spin coating system. Submicronic films were fabricated to make Lexan windows and to produce Lexan films coated on silicon targets. It was found that Lexan films can be produced with a range of desired thicknesses, from $230 \mathrm{~nm}$ down to $100 \mathrm{~nm}$, by altering the spin coating speed only. The characterization of Lexan thickness and surface analyses using AFM and interferometry showed high performance and reproducibility.

The total current measurement results of electron transport experiments with Lexan showed electron transport effects comparable to those of Quantomix ${ }^{\circledR}$ film. In wide spectrum experiments at the ESRF synchrotron, an MIC peak obtained from the Lexan layer resembled those measured previously for carbon layers. Using the preliminary MIC peak results for
Lexan, the authors obtained the following IMFP results: $8.5 \pm 3.0 \mathrm{~nm}$ at $6 \mathrm{keV}$, and $20.1 \pm 3.0 \mathrm{~nm}$ at 10 $\mathrm{keV}$.

This study suggests that Lexan films hold promise for future electron inelastic mean free path measurements.

\section{Acknowledgments}

This work was partially supported by Instituto de Ciencia de Materiales de Madrid under Proposal Number 25-02-828 in collaboration with Drs. G. R. Castro and J. Rubio-Zuazo from ESRF. The authors are grateful for the contributions of Dr. E. Tiferet and Dr. A. Givon to the experiments.

\section{References}

[1] Tanuma, S., Powell, C. J., and Penn, D. R. 2003. "Calculation of Electron Inelastic Mean Free Paths (IMFPs) VII. Reliability of the TPP-2M IMFP Predictive Equation.” Surface and Interface Analysis 35: 268-75.

[2] Powell, C. J., and Jablonski, A. 2010. "NIST Electron Inelastic-Mean-Free-Path Database.” NIST.

[3] Powell, C. J., and Jablonski, A. 1999. "Evaluation of Calculated and Measured Electron Inelastic Mean Free Paths Near Solid Surfaces.” J. Phys. Chem Ref. Data 28: 44.

[4] Werner, W. S. M. 2001. "Electron Transport in Solids for Quantitative Surface Analysis.” Surface and Interface Analysis 31: 141-76.

[5] Powell, C. J., and Jablonski, A. 2009. "Surface Sensitivity of X-Ray Photoelectron Spectroscopy." Nuclear Instruments and Methods in Physics Research Section A: Accelerators, Spectrometers, Detectors and Associated Equipment 601: 54-65.

[6] Sieghbahn, K. 1967. ESCA Atomic, Molecular and Solid State Structure Studied by Means of Electron Spectroscopy. Almqvist \& Wiksells, Uppsala.

[7] Powell, C. J. 1994, "Effects of Elastic and Inelastic Electron Scattering on Quantitative Surface Analyses by AES and XPS." Journal of Electron Spectroscopy and Related Phenomena 68: 605-16.

[8] Jablonski, A., and Powell, C. J. 1999. "Relationships between Electron Inelastic Mean Free Paths, Effective Attenuation Lengths, and Mean Escape Depths.” Journal of Electron Spectroscopy and Related Phenomena 100 (1999): 137-60.

[9] Sacchi, M. 2005. "Quantifying the Effective Attenuation 
Length in High-Energy Photoemission Experiments.” Physical Review B 71: 155117.

[10] Givon, A., Tiferet, E., Yaar, I., and Orion, I. 2010. "Measurements and Simulations of Electron Transport through Polystyrene and Polyimide.” 1st Bilateral Workshop of the JRC-IAEC Cooperation in Ein-Gedi-ISRAEL 3-6 May 2010.

[11] Givon, A. 2013. "Electron Transport in solids-Measuring Inelastic Mean Free Path in Energies of 5-15 keV.” Ph. D. Thesis, Ben- Gurion University.

[12] Givon, A., Tiferet, E., Castro, G. R. et al. 2013. "Hard $\mathrm{X}$-Ray Photoelectron Spectroscopy Study of Electron Spectral Structure beyond the Known Signal Electron Peak.” J. Chem. Chem. Eng. 7 (2013): 601-5.

[13] Givon, A., Tiferet, E., and Orion, I. 2012a. "Algorithm for Evaluating Layer Thickness Based on Electron Average Energy Shift Analysis.” Nuclear Instruments and Methods in Physics Research Section B: Beam Interactions with Materials and Atoms 288 (2012): 23-7 <doi:10.1016/j.nimb.2012.07.025>.

[14] Petty, M. C. 2011. “Organic Thin Film Deposition
Techniques.” Nanoscience and Nanotechnology 295-304. (www.aspbs.com/enn).

[15] Extrand, C. W. 1994. "Spin Coating of Very Thin Polymer Films.” Polymer Engineering and Science, 34 (1994), 390-394.

[16] Lawrence, C. J. 1988. "The Mechanics of Spin Coating of Polymer Films.” Physics of Fluids 31: 2786 $<$ doi:10.1063/1.866986>.

[17] Hall, D. B., Underhill, P., and Torkelson J. M. 1998. "Spin Coating." Polymer Engineering and Science 38: 2039-45.

[18] Rubio-Zuazo, J. and Castro, G. R. 2008a. "Information Depth Determination for Hard X-ray Photoelectron Spectroscopy up to $15 \mathrm{keV}$ Photoelectron Kinetic Energy.” Surface and Interface Analysis 40: 1438-43. $<$ doi:10.1002/SIA.2920>.

[19] Castro, G. R. 1998. "Optical Design of the General-Purpose Spanish X-ray Beamline for Absorption and Diffraction.” J. Synchrotron Rad. 5: 657.

[20] The Spanish CRG Beamline for Absorption and Diffraction. http://www.esrf.eu. 